\title{
Improved late gadolinium enhancement imaging of left ventricle with isotropic spatial resolution
}

\author{
Mehmet Akcakaya ${ }^{1 *}$, Hussein Rayatzadeh ${ }^{1}$, Susie Hong ${ }^{1}$, Thomas H Hauser ${ }^{1}$, Raymond H Chan ${ }^{1}$, Tamer A Basha ${ }^{1}$, \\ Kraig V Kissinger ${ }^{1}$, Beth Goddu', Warren J Manning ${ }^{1,2}$, Reza Nezafat ${ }^{1}$ \\ From 15th Annual SCMR Scientific Sessions \\ Orlando, FL, USA. 2-5 February 2012
}

\section{Background}

Recent studies have shown the prognostic value of the infarct border zone of late gadolinium enhancement (LGE) images in patients with myocardial infarction [1]. This border zone has also been associated with ventricular arrhythmia $[2,3]$. The accuracy of the characterization of this area depends on spatial resolution of the imaging. 3D LGE allows improved spatial resolution, especially in through-plane direction. However imaging with an isotropic spatial resolution necessitates very long scan time. In this study, we sought to investigate if compressed-sensing (CS) based image acceleration method [4] allows LGE imaging with isotropic spatial resolution.

\section{Methods}

A prospective random under-sampling LGE acquisition was implemented on 1.5T Philips scanner. A freebreathing ECG-triggered inversion-recovery GRE sequence with navigator-gating was used for all acquisitions on 18 patients ( 5 females, $52.8 \pm 16.3$ years) 10 to 20 minutes after bolus infusion of contrast agent. Each subject were imaged using two LGE sequence in random order: a) a 3-fold-accelerated LGE scan with isotropic spatial resolution of 1.2-to- $1.7 \mathrm{~mm}^{3}$, b) LGE scan with non-isotropic resolution of $1.7 \times 1.7 \times 4.0 \mathrm{~mm}^{3}$ were performed with imaging parameters of TR/TE $/ \alpha=5.2 /$ $2.6 \mathrm{~ms} / 25^{\circ}, \mathrm{FOV}=320 \times 320 \times 100 \mathrm{~mm}^{3}$. Random undersampling was implemented as described in [5], where the central k-space $(45 \times 35$ in ky-kz) was fully-sampled. Acquisition times were 3 mins assuming 100\% scan efficiency at $70 \mathrm{bpm}$ for both scans. The images from the

'Medicine, Beth Israel Deaconess Medical Center, Harvard Medical School, Boston, MA, USA

Full list of author information is available at the end of the article accelerated scans were reconstructed using an advanced CS-technique, called LOST [4].

\section{Results}

Figure 1 shows LGE images from a patient with hypertrophic cardiomyopathy acquired using two different approaches. An improved isotropic spatial resolution allows better characterization of the scar morphology. Figure 2 shows another example in a patient undergoing ICD implantation as a primary prevention of sudden cardiac death.

\section{Conclusions}

Accelerated LGE imaging with isotropic spatial resolution allows improved visualization of scar morphology. Further quantitative measurements of infarct border zones in a larger cohort of patients are needed to better understand the prognostic value of the improved scar imaging.

\section{Funding \\ NIH R01EB008743-01A2.}

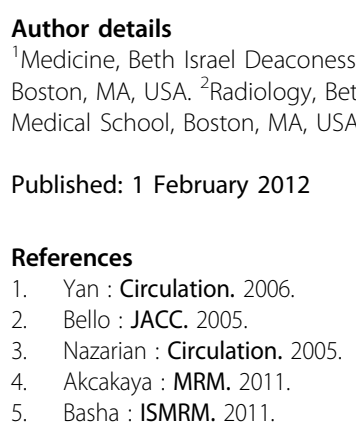
Boston, MA, USA. ${ }^{2}$ Radiology, Beth Israel Deaconess Medical Center, Harvard Medical School, Boston, MA, USA.

Published: 1 February 2012

References
1. Yan : Circulation. 2006.
2. Bello : JACC. 2005.
3. Nazarian : Circulation. 2005.
4. Akcakaya : MRM. 2011.
5. Basha : ISMRM. 2011.
Commons Attribution License (http://creativecommons.org/licenses/by/2.0), which permits unrestricted use, distribution, and reproduction in any medium, provided the original work is properly cited. 

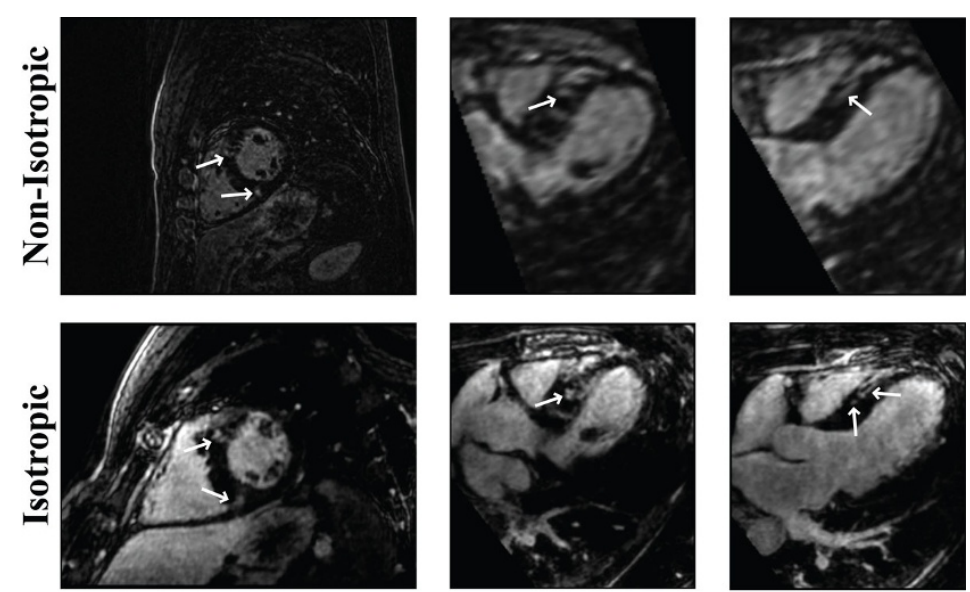

(a)

(b)

(c)

Figure 1 Reformatted LGE images from a patient with HCM, acquired using non-isotropic spatial resolution (top), and isotropic spatial resolution (bottom). An isotropic resolution allows better visualization of scar morphology in images acquired using LOST-accelerated acquisition.
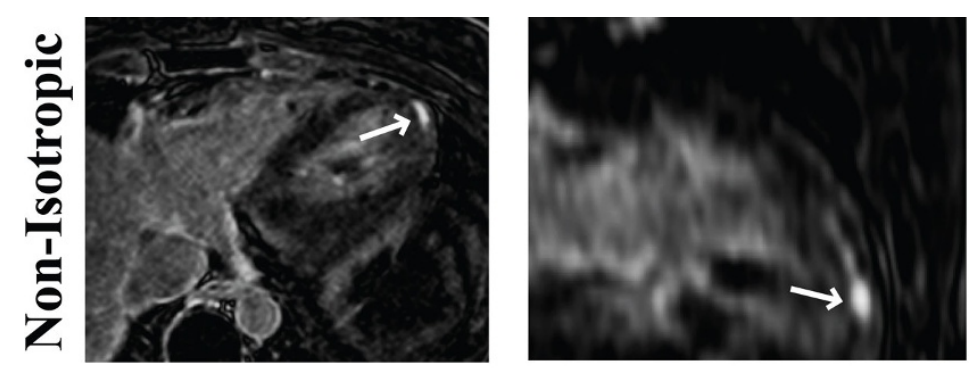

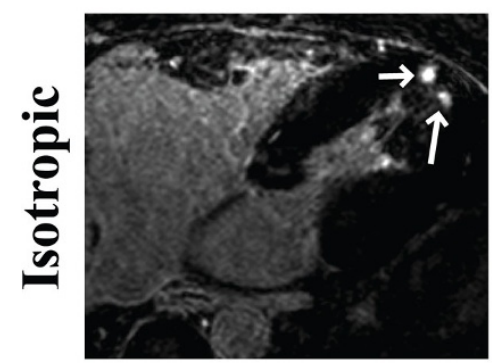

(a)

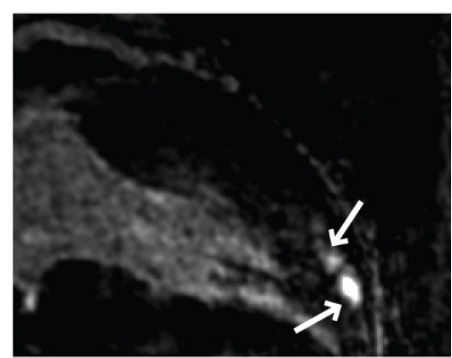

(b)

Figure 2 Axial (left) and reformatted long-axis (right) LGE images from a patient. Non-isotropic LGE was acquired with a spatial resolution $1.7 \times 1.7 \times 4.0 \mathrm{~mm}^{3}$ (top), whereas LOST-reconstructed isotropic resolution images from the accelerated scan had a resolution of $1.2 \times 1.2 \times 1.2 \mathrm{~mm}^{3}$ (bottom).

doi:10.1186/1532-429X-14-S1-O22

Cite this article as: Akcakaya et al:: Improved late gadolinium enhancement imaging of left ventricle with isotropic spatial resolution.

Journal of Cardiovascular Magnetic Resonance 2012 14(Suppl 1):O22.

\section{Submit your next manuscript to BioMed Central} and take full advantage of:

- Convenient online submission

- Thorough peer review

- No space constraints or color figure charges

- Immediate publication on acceptance

- Inclusion in PubMed, CAS, Scopus and Google Scholar

- Research which is freely available for redistribution 